\title{
MAPS ON IDEMPOTENT OPERATORS
}

\author{
PETER ŠEMRL \\ Department of Mathematics, University of Ljubljana \\ Jadranska 19, SI-1000 Ljubljana, Slovenia \\ E-mail: peter.semrl@fmf.uni-lj.si
}

\begin{abstract}
The set of all bounded linear idempotent operators on a Banach space $X$ is a poset with the partial order defined by $P \leq Q$ if $P Q=Q P=P$. Another natural relation on the set of idempotent operators is the orthogonality relation defined by $P \perp Q \Leftrightarrow P Q=Q P=0$. We briefly survey known theorems on maps on idempotents preserving order or orthogonality. We discuss some related results and open problems. The connections with physics, geometry, theory of automorphisms, and linear preserver problems will be explained. At the end we will prove a new result concerning bijective maps on idempotent operators preserving comparability.
\end{abstract}

1. Introduction. Throughout this paper $X$ will denote an infinite-dimensional real or complex Banach space and $\mathcal{B}(X)$ the algebra of all bounded linear operators on $X$. By $X^{\prime}$ we denote the dual of $X$. An operator $P \in \mathcal{B}(X)$ is called an idempotent operator if $P^{2}=P$. If $P$ is an idempotent, $P \notin\{0, I\}$, then the underlying Banach space $X$ can be decomposed into the direct $\operatorname{sum} X=\operatorname{Im} P \oplus \operatorname{Ker} P$, where $\operatorname{Im} P$ and $\operatorname{Ker} P$ denote the image and the kernel of $P$, respectively. Clearly, $P$ acts like the identity on $\operatorname{Im} P$, while the restriction of $P$ to Ker $P$ is the zero map. Thus, the matrix representation of $P$ with respect to the above direct sum decomposition of $X$ is

$$
P=\left[\begin{array}{ll}
I & 0 \\
0 & 0
\end{array}\right] .
$$

We denote by $I(X) \subset B(X)$ the set of all idempotent operators and by $I_{n}(X) \subset I(X)$ the subset of all idempotents of rank $n$, that is, the set of all idempotents whose image is an $n$-dimensional subspace of $X$. Here, $n$ is any positive integer.

We can define two natural relations on $I(X)$. It is easy to verify that $I(X)$ is a poset (partially ordered set) with the order defined by $P \leq Q$ if and only if $P Q=Q P=P$, $P, Q \in I(X)$. Of course, we have $P \leq Q$ if $P=0$ or $P=Q$ or $Q=I$. Assume that $P \leq Q$

2000 Mathematics Subject Classification: 46C05, 47B49.

Supported in part by a grant from the Ministry of Science of Slovenia.

The paper is in final form and no version of it will be published elsewhere. 
and that we do not have one of the trivial cases described in the previous sentence. Then $X=\operatorname{Im} P \oplus \operatorname{Im}(Q-P) \oplus \operatorname{Ker} Q$ and the matrix representations of $P$ and $Q$ with respect to this direct sum decomposition are

$$
P=\left[\begin{array}{lll}
I & 0 & 0 \\
0 & 0 & 0 \\
0 & 0 & 0
\end{array}\right] \text { and } Q=\left[\begin{array}{ccc}
I & 0 & 0 \\
0 & I & 0 \\
0 & 0 & 0
\end{array}\right] .
$$

Next, we define two idempotents $P, Q \in I(X)$ to be orthogonal if and only if $P Q=$ $Q P=0$. In this case we write $P \perp Q$. Clearly, for every pair $P, Q \in I(X)$ we have $P \perp Q$ if and only if $P+Q \in I(X)$. In this case we have $P, Q \leq P+Q$. Certainly, $P \perp Q$ if $P=0$ or $Q=0$ or $Q=I-P$. Assume that $P \perp Q$ and that we do not have one of the trivial cases described in the previous sentence. Then $X=\operatorname{Im} P \oplus \operatorname{Im} Q \oplus(\operatorname{Ker} Q \cap \operatorname{Ker} P)$ and the matrix representations of $P$ and $Q$ with respect to this direct sum decomposition are

$$
P=\left[\begin{array}{lll}
I & 0 & 0 \\
0 & 0 & 0 \\
0 & 0 & 0
\end{array}\right] \text { and } Q=\left[\begin{array}{ccc}
0 & 0 & 0 \\
0 & I & 0 \\
0 & 0 & 0
\end{array}\right] \text {. }
$$

These two relations are closely related. Namely, for any $P \in I(X)$ denote $P^{\perp}=\{Q \in$ $I(X): Q \perp P\}$. Let $P, Q$ be any idempotents. Then $P \leq Q$ if and only if $Q^{\perp} \subset P^{\perp}$.

Recall that an automorphism of a poset is a bijective map on this poset preserving order in both directions. Thus, an automorphism of the poset $I(X)$ is a bijective map $\phi: I(X) \rightarrow I(X)$ such that for every pair $P, Q \in I(X)$ we have $P \leq Q$ if and only if $\phi(P) \leq \phi(Q)$. If $\phi: I(X) \rightarrow I(X)$ preserves order in one direction only, that is, for every pair $P, Q \in I(X)$ we have $\phi(P) \leq \phi(Q)$ whenever $P \leq Q$, then $\phi$ is called monotone. Similarly, we say that $\phi: I(X) \rightarrow I(X)$ is an automorphism of $I(X)$ with respect to the orthogonality relation if $\phi$ is bijective and preserves orthogonality in both directions, that is, for every pair $P, Q \in I(X)$ we have $P \perp Q$ if and only if $\phi(P) \perp \phi(Q)$. We say that $\phi$ preserves orthogonality if for every pair $P, Q \in I(X)$ we have $\phi(P) \perp \phi(Q)$ whenever $P \perp Q$. Sometimes, when we want to emphasise that we do not assume that orthogonality is preserved in both directions, such a map is said to preserve orthogonality in one direction only.

Let $T \in B(X)$ be an invertible operator. Then, clearly, $P \mapsto T P T^{-1}, P \in I(X)$, is a bijective map preserving both order and orthogonality in both directions. In the complex case the same is true if $T: X \rightarrow X$ is a bounded invertible conjugate-linear operator. Similarly, if $T: X^{\prime} \rightarrow X$ is a bounded invertible linear or (in the complex case) conjugate-linear operator, then $P \mapsto T P^{\prime} T^{-1}, P \in I(X)$, is a bijective map preserving order and orthogonality in both directions. Here, $P^{\prime}$ denotes the adjoint of $P$. Such maps will be called standard maps on $I(X)$.

This paper is devoted to the problem of characterizing poset automorphisms of $I(X)$ and automorphisms of $I(X)$ with respect to the orthogonality relation. It turns out that every such automorphism is a standard map on $I(X)$. We will discuss possible improvements of this statement and related problems. In particular, if $P, Q \in I(X)$ satisfy $P \leq Q$ or $P \perp Q$, then $P$ and $Q$ commute. So, we have here a related problem of characterizing bijective maps on $I(X)$ preserving commutativity (in both directions). Of course, we say that $\phi: I(X) \rightarrow I(X)$ preserves commutativity if $\phi(P) \phi(Q)=\phi(Q) \phi(P)$ 
whenever $P Q=Q P, P, Q \in I(X)$, and it preserves commutativity in both directions if for every pair of idempotents $P, Q \in I(X)$ we have $\phi(P) \phi(Q)=\phi(Q) \phi(P)$ if and only if $P Q=Q P$. There exist bijective maps on $I(X)$ preserving commutativity in both directions that are not of the standard form. Indeed, let $\tau: I(X) \rightarrow\{0,1\}$ be a map with the property that $\tau(P)=1 \Leftrightarrow \tau(I-P)=1, P \in I(X)$. Define $\xi: I(X) \rightarrow I(X)$ by $\xi(P)=\tau(P) P+(1-\tau(P))(I-P), P \in I(X)$. We will call every such map an ortho-permutation on $I(X)$. For every $P \in I(X)$ it either maps both $P$ and $I-P$ into themselves, or into each other. It is a bijective map preserving commutativity in both directions. So, the natural question here is whether every bijective map on $I(X)$ preserving commutativity in both directions is a standard map composed with an orthopermutation. Is this true under the weaker assumption of preserving commutativity in one direction only?

If $X=H$ is a Hilbert space then it is natural to consider order or orthogonality preserving maps not only on the set of all idempotents $I(H)$, but also on the subset $P(H)$ consisting of all projections, that is, selfadjoint idempotent operators. If we associate to each projection its image then we obtain a bijective correspondence between $P(H)$ and the lattice of closed subspaces of $H$. The problem of characterizing bijective maps on the set of all projections preserving order in both directions becomes via this identification the problem of characterizing automorphisms of the lattice of closed subspaces of $H$. This problem was solved by Fillmore and Longstaff [5] even for general Banach spaces. The problem of characterizing bijective maps on $P(H)$ preserving orthogonality in both directions is important because of applications in physics. In fact, because of these applications, it is even more interesting to study orthogonality preserving maps defined not on the whole set $P(H)$, but on the subset of all projections of a given fixed (finite or infinite) rank. And finally, this kind of problems have been recently studied also in the more general case when the underlying space $H$ is an indefinite inner product space.

The above described problems are interesting by themselves but they are important also because of many applications. In the next section we will point out the connections with physics, geometry, theory of automorphisms, and linear preserver problems. The third section will be devoted to the statement of known results and open problems. We will conclude the paper with a new result. Ovchinnikov [19] characterized bijective maps on $I(X)$ preserving order in both directions. He posed the problem whether we can get a nice structural result under the weaker assumption that the comparability of idempotents is preserved in both directions. The last section will be devoted to the solution of this problem. At the end we will give a list of references containing also some items that will not be cited in the paper. Nevertheless, we decided to include them as they are so closely related to the problems treated in the paper.

2. Motivation. One of the important problems in foundations of quantum mechanics is the description of probability measures on the set of experimentally verifiable propositions that form an orthomodular poset. The remarkable Gleason's theorem [6] characterizes countably additive probability measures on the set $P(H)$ of all projections on a Hilbert space $H$. To extend this result to other topological linear spaces we have to deal with 
idempotents instead of with projections. A theory of signed measures on the poset $I(H)$ (or more generally, a poset of all idempotents in a von Neumann algebra) was developed by Matveichuk and Mushtari (see [4] and the references therein). This theory was based on the observation that every $P \in I(H)$ is selfadjoint with respect to some inner product $(x, y)_{A}=(A x, y)$ on $H$. Here, $A$ is an invertible positive operator. For example, we can take $A$ to be $A=P^{*} P+\left(I-P^{*}\right)(I-P)$. To each strictly positive $A$ we associate the set $\Pi_{A}$ consisting of all idempotents $P \in I(H)$ being selfadjoint with respect to $(\cdot, \cdot)_{A}$. The poset $I(H)$ is the union of such sets and on each of these sets we can apply the classical Gleason's theorem when studying measure theory on $I(H)$. To apply Gleason's theorem the assumption that the measure under the consideration is bounded on every $\Pi_{A}$ is needed. This condition seems to be non-internal, and thus, the question here is, whether the family of all sets $\Pi_{A}$, where $A$ is any invertible positive operator, can be defined in terms of $\leq$ alone. This problem motivated Ovchinnikov to characterize poset automorphisms of $I(H)$ (see [4, Appendix, p. 84-86] and [19, Remark 4.2]).

Recently, Ovchinnikov's result proved to be useful in the study of another classical result in quantum mechanics, that is, Wigner's unitary-antiunitary theorem formulated in [30] and proved in $[3,10]$ (for some recent results on this theorem we refer to [11, $12,13,14,15])$ stating that every quantum mechanical invariance transformation can be represented by a unitary or an antiunitary operator on a complex Hilbert space. Uhlhorn [29] extended Wigner's theorem by characterizing transformations preserving only the logical structure of the quantum mechanical system and not the complete probabilistic structure. The reformulation in the mathematical language states that every bijective map on the set of all rank one projections on a Hilbert space $H, \operatorname{dim} H \geq 3$, preserving orthogonality in both directions is induced by a unitary or an antiunitary operator on $H$. Recently, this was generalized in two directions: to the set of all projections of a given fixed rank [16] and to indefinite inner product spaces [18]. The original proof of the second extension was based on the result of Ovchinnikov. Later, a shorter proof was found $[23,26]$ based on the characterization of bijective orthogonality preserving (zero product preserving) maps on rank one idempotent operators.

The results on order or orthogonality preserving maps on idempotents have applications also in pure mathematics. Let $X$ be an infinite-dimensional Banach space. It is well-known that every automorphism (linear bijective multiplicative map) of the algebra $B(X)$ is inner, that is, of the form $A \mapsto T A T^{-1}, A \in B(X)$. Here, $T$ is an invertible bounded linear operator. A more general problem is to consider bijective maps on $B(X)$ that are assumed to be only multiplicative (in this case we are interested in the automorphisms of the multiplicative semigroup $B(X)$ ). It turns out [21] that such maps are again of the form $A \mapsto T A T^{-1}, A \in B(X)$, with the only difference that in this more general setting $T$ is either linear, or (in the complex case) conjugate-linear. This statement follows easily from the structural results on order or orthogonality preserving maps (see [23]). Namely, it is obvious that $I(X)$ is invariant under every multiplicative map on $B(X)$ and the restriction of every such map to $I(X)$ preserves both order and orthogonality.

Observe further that the restriction of every linear idempotent preserving map on $B(X)$ to $I(X)$ preserves both order and orthogonality. Indeed, this follows easily from 
the facts that for $P, Q \in I(X)$ we have $P \leq Q \Leftrightarrow Q-P \in I(X)$ and $P \perp Q \Leftrightarrow P+Q \in$ $I(X)$. So, the characterization of linear maps on $B(X)$ preserving idempotents in both directions can be easily deduced from the results on order or orthogonality preservers on $I(X)$. Recently, many linear preserver problems were solved by reducing them to the problem of characterizing linear maps preserving idempotent operators [7]. Perhaps the most important linear preserver result obtained in this way is the Aupetit's solution [2] of Kaplansky's problem of characterizing linear invertibility preserving maps for von Neumann algebras. Observe that when reducing a certain linear preserver problem first to the problem of characterizing linear maps preserving idempotents, and then to the structural problem for order preserving or orthogonality preserving maps on idempotents, we finally arrive at a non-linear problem. This may result in a stronger conclusion than one expected at the beginning. For example, Araujo and Jarosz [1] obtained a characterization of linear biseparating maps (maps preserving zero products) on $B(X)$. Using the above suggested approach a non-linear extension of their result was given in [25].

In this paper we will restrict our attention to transformations on the sets of idempotent operators acting on infinite-dimensional spaces. Similar problems are, of course, interesting also in the finite-dimensional case. The finite-dimensional case is on one hand easier, but on the other hand, under the finite-dimensionality condition we want to have structural results for order and orthogonality preserving maps under much weaker assumptions, and so, some finite-dimensional problems are even more difficult. Also, some of them require a different approach. An interested reader can find results on the finitedimensional case in [27]. Let us just mention that these results are closely connected to the geometry of matrices and to the geometry of Grassmann spaces (see [28]).

3. Results and open problems. The main results that we would like to present and then discuss possible improvements are the characterizations of automorphisms of $I(X)$ with respect to order or orthogonality relation. They both follow from the following result proved in [25].

THEOREM 3.1. Let $X$ be an infinite-dimensional real or complex Banach space and $\phi$ : $I(X) \rightarrow I(X)$ a bijective map preserving commutativity in both directions. Then there exist an ortho-permutation $\xi: I(X) \rightarrow I(X)$ and either a bounded invertible linear or (in the complex case) conjugate-linear operator $T: X \rightarrow X$ such that

$$
\phi(P)=T \xi(P) T^{-1}, \quad P \in I(X),
$$

or a bounded invertible linear or (in the complex case) conjugate-linear operator $T: X^{\prime} \rightarrow$ $X$ such that

$$
\phi(P)=T \xi(P)^{\prime} T^{-1}, \quad P \in I(X) .
$$

In the second case $X$ must be reflexive.

The proof of this theorem has been inspired by some ideas that can be found in the paper of Rickart [20]. He considered automorphisms of the general linear group on infinitedimensional spaces. Such automorphisms preserve involutions (operators $J$ satisfying $J^{2}=I$ ) and their restrictions to the set of involutions preserve commutativity. Involutions and idempotents are closely related (every involution is of the form $I-2 P$, where $I$ is the 
identity operator and $P$ is an idempotent). The first few steps of the proof of the above result are based on this simple observation and some ideas from [20].

Assume now that $\phi: I(X) \rightarrow I(X)$ is a bijective map preserving orthogonality in both directions. Then one can prove that it preserves commutativity in both directions. The main idea is the following. Assume that $P_{1} \in I(X)$ and $P_{2} \in I(X)$ commute. Then there exists a direct sum decomposition of $X$ such that with respect to this direct sum decomposition $P_{1}$ and $P_{2}$ have the following matrix representations:

$$
P_{1}=\left[\begin{array}{llll}
I & 0 & 0 & 0 \\
0 & 0 & 0 & 0 \\
0 & 0 & I & 0 \\
0 & 0 & 0 & 0
\end{array}\right] \text { and } P_{2}=\left[\begin{array}{cccc}
0 & 0 & 0 & 0 \\
0 & I & 0 & 0 \\
0 & 0 & I & 0 \\
0 & 0 & 0 & 0
\end{array}\right] \text {. }
$$

Here, some rows and columns may be absent. Thus, it is easy to see that the idempotents $Q_{1}=P_{1}\left(I-P_{2}\right), Q_{2}=P_{2}\left(I-P_{1}\right), Q_{3}=P_{1} P_{2}$, and $Q_{4}=I-P_{1}-P_{2}+P_{1} P_{2}$ are orthogonal and $Q_{1}+Q_{2}+Q_{3}+Q_{4}=I$. Using the bijectivity and the orthogonality preserving assumptions it is possible to show that $\phi\left(Q_{j}\right), j=1,2,3,4$, are pairwise orthogonal idempotents whose sum is the identity. Moreover, the bijectivity and the orthogonality preserving property yield that $\phi\left(P_{1}\right)=\phi\left(Q_{1}\right)+\phi\left(Q_{3}\right)$ and $\phi\left(P_{2}\right)=\phi\left(Q_{2}\right)+\phi\left(Q_{3}\right)$, and thus, $\phi\left(P_{1}\right)$ and $\phi\left(P_{2}\right)$ commute. In the same way we see that the inverse of $\phi$ preserves commutativity. It is then easy to deduce the following characterization of $\perp$ automorphisms from the above result.

Corollary 3.2. Let $X$ be an infinite-dimensional real or complex Banach space and $\phi: I(X) \rightarrow I(X)$ a bijective map preserving orthogonality in both directions. Then there exists either a bounded invertible linear or (in the complex case) conjugate-linear operator $T: X \rightarrow X$ such that

$$
\phi(P)=T P T^{-1}, \quad P \in I(X),
$$

or a bounded invertible linear or (in the complex case) conjugate-linear operator $T: X^{\prime} \rightarrow$ $X$ such that

$$
\phi(P)=T P^{\prime} T^{-1}, \quad P \in I(X) .
$$

In the second case $X$ must be reflexive.

We have already observed that $\perp$ and $\leq$ are closely related relations. In particular, it can be proved that a bijective map on $I(X)$ preserves order in both directions if and only if it preserves orthogonality in both directions [25, Proposition 1.1]. Therefore, Ovchinnikov's result [19] follows directly from the characterization of $\perp$-automorphisms.

Corollary 3.3. Let $X$ be an infinite-dimensional real or complex Banach space and $\phi$ a poset automorphism of $I(X)$. Then there exists either a bounded invertible linear or (in the complex case) conjugate-linear operator $T: X \rightarrow X$ such that

$$
\phi(P)=T P T^{-1}, \quad P \in I(X),
$$

or a bounded invertible linear or (in the complex case) conjugate-linear operator $T: X^{\prime} \rightarrow$ $X$ such that

$$
\phi(P)=T P^{\prime} T^{-1}, \quad P \in I(X) .
$$

In the second case $X$ must be reflexive. 
Let us now discuss possible improvements. The main open problem here is whether we can get the same conclusion as in the above two corollaries under the weaker assumption that the orthogonality (order) is preserved in one direction only. Even a much simpler problem, that is, the question whether every bijective map on $I(X)$ preserving both orthogonality and order in one direction only must be standard, has not been solved yet.

What about the bijectivity assumption? It is easy to find examples of "wild" injective maps preserving orthogonality or order in both directions. Indeed, let $H$ be an infinitedimensional Hilbert space. Then $H$ is isomorphic to $H \oplus H$. So, every map acting on $I(H)$ can be considered as a map from $I(H)$ into $I(H \oplus H)$. Let $\varphi: I(H) \rightarrow I(H)$ be any order preserving map. For example, we can construct such a map in the following way. We choose any map $\varphi_{1}: I_{1}(H) \rightarrow I(H)$. For every rank two idempotent $Q \in I_{2}(H)$ the set $M_{Q}=\left\{R \in I(H): \varphi_{1}(P) \leq R\right.$ for every $P \in I_{1}(H)$ satisfying $\left.P \leq Q\right\}$ is nonempty as it necessarily contains $I$. So we can choose a map $\varphi_{2}: I_{2}(H) \rightarrow I(H)$ with the property that $\varphi_{2}(Q) \in M_{Q}$ for every $Q \in I_{2}(H)$. Clearly, the map $\varphi: I(H) \rightarrow I(H)$ defined by $\varphi(0)=0, \varphi(P)=\varphi_{1}(P)$ if $P \in I_{1}(H), \varphi(P)=\varphi_{2}(P)$ if $P \in I_{2}(H)$, and $\varphi(P)=I$ otherwise, preserves order. Then a map $\phi: I(H) \rightarrow I(H \oplus H)$ defined by

$$
\phi(P)=\left[\begin{array}{cc}
\varphi(P) & 0 \\
0 & P
\end{array}\right], \quad P \in I(H),
$$

is an injective map preserving order in both directions which is far from being of a standard form. A similar construction gives "wild" injective maps preserving orthogonality in both directions. For example we can choose $\varphi: I(H) \rightarrow I(H)$ to be any map satisfying $\varphi(P) \leq P$ for every $P \in I(H)$.

Hence, at least the surjectivity assumption is essential in the above results. We next give non-standard examples of surjective maps preserving order or orthogonality in one direction only. Let $H$ be a separable Hilbert space with orthonormal basis $\left\{e_{j}: j=\right.$ $1,2, \ldots\}$. Let $T: H \rightarrow H$ be a shift operator, $T e_{j}=e_{j+1}, j=1,2, \ldots$ Then $T^{*} T=I$, while $T T^{*}$ is a projection onto the closed linear span of $e_{2}, e_{3}, \ldots$ Denote by $S$ the set of all $P \in I(H)$ mapping $e_{1}$ into 0 and leaving the orthogonal complement of $e_{1}$ invariant. Define $\phi: I(H) \rightarrow I(H)$ by $\phi(P)=T^{*} P T$ for every $P \in S$ and $\phi(P)=0$ otherwise. Then this is a surjective map preserving orthogonality in one direction. And the map $\phi: I(H) \rightarrow I(H)$ defined by $\phi(P)=T^{*} P T$ for every $P \in S$ and $\phi(P)=I$ otherwise is an example of a surjective monotone map.

So, the weak preserving property (in one direction only) together with the surjectivity assumption does not imply that the maps under consideration are standard. It remains to answer the question whether we get nice structural results if we assume surjectivity together with the strong preserving property. It is easy to give the affirmative answer. Indeed, we will show that Corollary 3.2 holds true without the injectivity assumption. Thus, assume that $\phi: I(X) \rightarrow I(X)$ is a surjective map preserving orthogonality in both directions. We have to show that then $\phi$ is bijective. Assume on the contrary that there are $P, Q \in I(X)$ with $P \neq Q$ and $\phi(P)=\phi(Q)$. Because $P \neq Q$ we can find $R \in I(S)$ such that either $R \perp P$ and $R \not \perp Q$, or $R \perp Q$ and $R \not \perp P$. We will consider only the first case. Then $\phi(R) \perp \phi(P)=\phi(Q) \not \perp \phi(R)$, a contradiction. In almost the same way we 
show that Corollary 3.3 holds true if we replace the bijectivity assumption by the weaker surjectivity assumption.

In the rest of this section we will describe some other known results characterizing monotone or orthogonality preserving maps on some sets of idempotents acting on infinite-dimensional spaces. In all of these results we deal with bijective maps having the strong preserving property, that is, we always assume that either order, or orthogonality is preserved in both directions. So, in exactly the same way as above we can ask whether each of these results can be improved by replacing the strong preserving property by a weaker assumption that the considered relation (order or orthogonality) is preserved in one direction only.

Let $X$ be a real or complex infinite-dimensional Banach space. In [25] we have considered orthogonality preserving maps defined not only on the whole set $I(X)$, but also orthogonality preserving maps defined on the subset $I_{n}(X) \subset I(X)$ consisting of all idempotents of rank $n$. Here, $n$ is a fixed positive integer. It was proved that every bijective map on $I_{n}(X)$ preserving orthogonality in both directions is of a standard form. We conjecture that (at least when $X$ is a Hilbert space) we have the same conclusion if we replace $I_{n}(X)$ by $I_{\infty}(X)$. Here, $I_{\infty}(X) \subset I(X)$ denotes the subset of all idempotents whose image and kernel are both infinite-dimensional. Let us explain why we need such a definition of $I_{\infty}(X)$. The other possibility would be to consider orthogonality preserving bijective maps on $I^{\infty}(X)$ with $I^{\infty}(X)$ being defined as the set of all idempotents with infinite-dimensional images. Clearly, $I_{\infty}(X) \subset I^{\infty}(X)$. Let $\varphi: I^{\infty}(X) \backslash I_{\infty}(X) \rightarrow I^{\infty}(X) \backslash I_{\infty}(X)$ be any bijective map. Then the bijective map $\phi: I^{\infty}(X) \rightarrow I^{\infty}(X)$ defined by $\phi(P)=P$ if $P \in I_{\infty}(X)$ and $\phi(P)=\varphi(P)$ if $P \in I^{\infty}(X) \backslash I_{\infty}(X)$ preserves orthogonality in both directions. So, we cannot say anything about the behaviour of bijective maps $\phi: I^{\infty}(X) \rightarrow I^{\infty}(X)$ preserving orthogonality in both directions on the subset $I^{\infty}(X) \backslash I_{\infty}(X)$.

Let now $H$ be an infinite-dimensional real or complex Hilbert space. Then we are interested not only in maps on the set of all bounded linear idempotent operators $I(H)$, but also in maps acting on the sets of projections. Györy [8] and Šemrl [24] independently characterized bijective maps on $P_{n}(H)$ or $P_{\infty}(H)$ preserving orthogonality in both directions. Here, $n$ is any positive integer (the special case $n=1$ is known as Uhlhorn's theorem) and $P_{n}(H) \subset P(H)$ denotes the set of all projections on $H$ of rank $n$, while $P_{\infty}(H)$ denotes the set of all projections with infinite rank and corank. All such maps are of the expected form $P \mapsto U P U^{*}$, where $U$ is a unitary or an antiunitary operator. It follows easily that every bijective map $\phi: P(H) \rightarrow P(H)$ preserving orthogonality in both directions is of the same form. Indeed, 0 is the only projection orthogonal to every other projection. Thus, $\phi(0)=0$. Among nonzero projections we can characterize rank one projections as those projections $P$ having maximal orthocomplement $P^{\perp}=\{Q \in P(H): Q \perp P\}$. Thus, $\phi$ maps $P_{1}(H)$ onto itself. Now we can apply the above mentioned result of Uhlhorn to conclude that the restriction of $\phi$ to $P_{1}(H)$ is of the form $P \mapsto U P U^{*}, P \in P_{1}(H)$, where $U$ is a unitary or an antiunitary operator. After composing $\phi$ with the map $P \mapsto U^{*} P U, P \in P(H)$, we may assume that $\phi(P)=P$ for every rank one projection $P$. It follows that for every $Q \in P(H)$ the set of all rank 
one projections $P$ orthogonal to $Q$ is the same as the set of all rank one projections $P$ orthogonal to $\phi(Q)$, and consequently, $\phi(Q)=Q$ for every $Q \in P(H)$, as desired.

The next natural problem here is the characterization of bijective maps on $P(H)$ preserving order in both directions. Of course, we can identify projections with closed subspaces of $H$. Thus, we are asking about the general form of automorphisms of the lattice of closed subspaces of $H$. This problem has been solved even for general Banach spaces $X$ by Fillmore and Longstaff [5]. They proved that every such lattice automorphism is induced by a bounded invertible linear or (in the complex case) conjugate-linear operator acting on $X$. It should be mentioned here that bijective maps on the lattice of closed subspaces of $H$ preserving order in both directions do not necessarily preserve orthogonality, or equivalently, they are not necessarily induced by a unitary or an antiunitary operator.

The analogue of Ovchinnikov's theorem for quaternionic Hilbert spaces was proved in [27]. Let us conclude with a remark on indefinite inner product spaces. In the case of Hilbert spaces Uhlhorn's theorem characterizes bijective maps on the set of all rank one projections preserving orthogonality in both directions, or equivalently, it characterizes bijective maps on the set of all one-dimensional spaces preserving orthogonality in both directions. As mentioned above Uhlhorn's result has been generalized to maps acting on the set of all closed subspaces of a given fixed (finite or infinite) rank. So far, only Uhlhorn's theorem has been generalized to the more general case when the underlying space is an indefinite inner product space [18], while the problem for higher dimensional spaces remains open.

4. Comparability preserving maps. This section is devoted to a new result which extends the Ovchinnikov's characterization of automorphisms of the poset of idempotent operators on a Banach space $X$. He posed the problem of describing the general form of bijective maps on $I(X)$ preserving comparability in both directions. More precisely, for $P, Q \in I(X)$ we write $P \sim Q$ if $P \leq Q$ or $Q \leq P$. A map $\phi: I(X) \rightarrow I(X)$ preserves comparability in both directions if for every pair $P, Q \in I(X)$ we have $P \sim Q$ if and only if $\phi(P) \sim \phi(Q)$. A map $\phi: I(X) \rightarrow I(X)$ is said to be an anti-automorphism if it is bijective and if for every pair $P, Q \in I(X)$ we have $P \leq Q$ if and only if $\phi(P) \geq \phi(Q)$. Clearly, a map $\phi$ is an anti-automorphism of the poset $I(X)$ if and only if the map $P \mapsto \phi(I-P), P \in I(X)$, is an automorphism. So, the description of the general form of anti-automorphisms of $I(X)$ follows directly from Ovchinnikov's characterization of automorphisms of $I(X)$. Both automorphisms and anti-automorphisms are bijective maps preserving comparability in both directions. A map $\eta: I(X) \rightarrow I(X)$ defined by $\eta(0)=I$, $\eta(I)=0$, and $\eta(P)=P$ for every $P \in I(X) \backslash\{0, I\}$ is another example of a bijective map preserving comparability in both directions. Further, any composition of bijective maps preserving comparability in both directions is again a map of this type. We will show that we have exhausted all examples. Throughout this paper we have considered only the case when the underlying spaces are infinite-dimensional. We will make here an exception by considering both the finite and the infinite-dimensional case. However, we expect that the finite-dimensional case can be substantially improved. To explain possible improvements 
we would need the notion of an injective degenerate order preserving map [27, 28]. As the emphasis in this paper is on the infinite-dimensional case we omit the details here.

Theorem 4.1. Let $X$ be a real or complex Banach space, $\operatorname{dim} X \geq 3$, and $\phi: I(X) \rightarrow$ $I(X)$ a bijective map preserving comparability in both directions. Then either $\phi$ is an automorphism of the poset $I(X)$, or an anti-automorphism of the poset $I(X)$, or $\phi=\psi \circ \eta$, where $\psi$ is an automorphism of the poset $I(X)$, or $\phi=\psi \circ \eta$, where $\psi$ is an antiautomorphism of the poset $I(X)$.

Proof. There are exactly two idempotents that are comparable with all idempotents, namely, 0 and $I$. Thus, $\phi(\{0, I\})=\{0, I\}$. Denote $J(X)=I(X) \backslash\{0, I\}$. Then the restriction of $\phi$ to $J(X)$ is a bijective map onto $J(X)$ and we have to prove that it is either an automorphism, or an anti-automorphism.

To each $P \in J(X)$ we associate a subset $P^{\sim} \subset J(X)$ defined by $P^{\sim}=\{Q \in J(X)$ : $P \sim Q\}$. Clearly, $P^{\sim}=P_{\leq} \cup P_{\geq}$, where $P_{\leq}=\{Q \in J(X): Q \leq P\}$ and $P_{\geq}=\{Q \in$ $J(X): P \leq Q\}$.

In the next step we will prove that if $P^{\sim}=\mathcal{A} \cup \mathcal{B}$ for some $\mathcal{A}, \mathcal{B} \subset J(X)$ satisfying $\mathcal{A} \cap \mathcal{B}=\{P\}$ and $R \sim Q$ for every $R \in \mathcal{A}$ and every $Q \in \mathcal{B}$, then either

- $\mathcal{A}=P_{\leq}$and $\mathcal{B}=P_{\geq}$, or

- $\mathcal{A}=P_{\geq}$and $\mathcal{B}=P_{\leq}$, or

- $\mathcal{A}=\{P\}$ and $\mathcal{B}=P^{\sim}$, or

- $\mathcal{A}=P^{\sim}$ and $\mathcal{B}=\{P\}$.

Indeed, there is nothing to prove if $\mathcal{A}=\{P\}$ or $\mathcal{B}=\{P\}$. So assume that $\mathcal{A} \neq\{P\}$ and $\mathcal{B} \neq\{P\}$. Then we have to prove that one of these two sets consists of all nonzero idempotents that are below $P$ and the other one of all idempotents $\neq I$ that are above $P$. Assume on the contrary that there are idempotents $R \in \mathcal{A}$ and $Q \in \mathcal{B}, R \neq P$ and $Q \neq P$, that are both below $P$ or both above $P$. We will consider only the first possibility. By our assumptions, $R$ and $Q$ are comparable, and thus, we have either $Q \leq R \leq P$, or $R \leq Q \leq P$. Once again, we will consider only the first possibility. As the image of $R$ is a proper subspace of the image of $P$ we can find a nonzero bounded linear functional $f$ on $\operatorname{Im} P$ such that $f(\operatorname{Im} R)=0$. We extend $f$ to a bounded linear functional on $X$ by setting $f(x)=0$ for every $x \in \operatorname{Ker} P$. We further choose a nonzero $x \in \operatorname{Im} Q$ and denote by $x \otimes f$ a bounded linear rank one operator on $X$ defined by $(x \otimes f) z=f(z) x, z \in X$. It is trivial to check that $Q+x \otimes f \leq P, Q(Q+x \otimes f)=R(Q+x \otimes f)=Q+x \otimes f$, and $(Q+x \otimes f) Q=(Q+x \otimes f) R=Q$. Thus, $Q+x \otimes f$ is comparable with $P$. As it is not comparable with $Q \in \mathcal{B}$, it does not belong to $\mathcal{A}$, and similarly, $Q+x \otimes f \notin \mathcal{B}$, a contradiction.

Our assumption that $\phi$ preserves comparability in both directions together with the previous statement yield that for every $P \in J(X)$ we have $\phi\left(P^{\sim}\right)=(\phi(P))^{\sim}$ and either $\phi\left(P_{\leq}\right)=\phi(P)_{\leq}$and $\phi\left(P_{\geq}\right)=\phi(P)_{\geq}$, or $\phi\left(P_{\leq}\right)=\phi(P)_{\geq}$and $\phi\left(P_{\geq}\right)=\phi(P)_{\leq}$. An idempotent $P$ is of rank one or corank one if and only if one of the sets $P_{\leq}$and $P_{\geq}$is singleton $\{P\}$. Thus, $\phi$ maps the set of all idempotents of rank one or corank one onto itself. We will call every idempotent of rank one or corank one extremal. 
We may assume with no loss of generality that there is an idempotent of rank one that is mapped by $\phi$ into an idempotent of rank one since otherwise we replace $\phi$ by the map $P \mapsto \phi(I-P)$. Denote this idempotent by $P_{0}$.

Next observe that if $P$ and $Q, P \neq Q$, are extremal idempotents and $P \sim Q$ then one of $P, Q$ must be of rank one and the other one of corank one.

Let $P$ be any idempotent of rank one. Assume first that $\operatorname{dim} X \geq 4$. Then we can find $Q$ of corank one such that $P \leq Q$ and $P_{0} \leq Q$. It follows that $\phi(P) \sim \phi(Q)$ and $\phi\left(P_{0}\right) \sim \phi(Q)$. Thus, $\phi(P)$ is of rank one as well. Thus, $\phi$ preserves idempotents of rank one when $\operatorname{dim} X \geq 4$. If $\operatorname{dim} X=3$, then elementary linear algebra arguments (we identify operators with matrices) yield the existence of an idempotent $P_{1}$ of rank one and idempotents $Q_{1}, Q_{2}$ of rank two such that $P_{0} \leq Q_{1} \geq P_{1}$ and $P_{1} \leq Q_{2} \geq P$. Indeed, there is nothing to prove if $P=P_{0}$. So, assume that $P \neq P_{0}$. If $P$ and $P_{0}$ have the same image, then, after applying a similarity transformation, we may assume that

$$
P=\left[\begin{array}{lll}
1 & 0 & 0 \\
0 & 0 & 0 \\
0 & 0 & 0
\end{array}\right] \text { and } P_{0}=\left[\begin{array}{lll}
1 & 1 & 0 \\
0 & 0 & 0 \\
0 & 0 & 0
\end{array}\right]
$$

and so the choice $P_{1}=P, Q_{1}=Q_{2}=E_{11}+E_{22}$ works. Here, $E_{i j}$ denotes the usual matrix unit. In the case that the images of $P$ and $P_{0}$ are not the same, we may assume after replacing them by simultaneously similar matrices that

$$
P=\left[\begin{array}{lll}
1 & 0 & 0 \\
0 & 0 & 0 \\
0 & 0 & 0
\end{array}\right] \text { and } P_{0}=\left[\begin{array}{ccc}
x w & y w & z w \\
x & y & z \\
0 & 0 & 0
\end{array}\right]
$$

for some scalars $x, y, z, w$ with $y+x w=1$. In this case we set

$$
P_{1}=\left[\begin{array}{ccc}
x w & y w & 0 \\
x & y & 0 \\
0 & 0 & 0
\end{array}\right] \text { and } Q_{2}=\left[\begin{array}{lll}
1 & 0 & 0 \\
0 & 1 & 0 \\
0 & 0 & 0
\end{array}\right]
$$

Then $P_{1} \leq Q_{2} \geq P$. As $P_{0}$ and $P_{1}$ have the same image we can find by the previous step a rank two idempotent $Q_{1}$ satisfying $P_{0} \leq Q_{1} \geq P_{1}$, as desired.

Now, as $\phi\left(P_{0}\right)$ is of rank one, $\phi\left(Q_{1}\right)$ has to be of rank two which further yields that $\phi\left(P_{1}\right)$ is of rank one. Consequently, $\phi\left(Q_{2}\right)$ is of rank two, and thus, $\phi(P)$ is of rank one, as desired.

We will complete the proof by showing that for every $P, Q \in J(X)$ the inequality $P \leq Q$ holds true if and only if $\phi(P) \leq \phi(Q)$. All we have to show is that for any $P \in I(X)$ the set of all idempotents of rank one that are below $P$ are mapped onto the set of all idempotents of rank one that are below $\phi(P)$. As $\phi$ and its inverse have the same properties it is enough to show that if $R$ is of rank one and $R \leq P$ then $\phi(R) \leq \phi(P)$. From $R \leq P$ it follows that $\phi(R) \sim \phi(P)$ and because $\phi(R)$ is of rank one we have actually $\phi(R) \leq \phi(P)$. This completes the proof. 


\section{References}

[1] J. Araujo and K. Jarosz, Biseparating maps between operator algebras, J. Math. Anal. Appl. 282 (2003), 48-55.

[2] B. Aupetit, Spectrum-preserving linear mappings between Banach algebras or JordanBanach algebras, J. London Math. Soc. 62 (2000), 917-924.

[3] V. Bargmann, Note on Wigner's theorem on symmetry operations, J. Math. Phys. 5 (1964), 862-868.

[4] S. V. Dorofeev et al., Measures on projections and orthomodular posets, in: Collection of papers, Kazan Tracts in Mathematics 4 (1998), 1-114.

[5] P. A. Fillmore and W. E. Longstaff, On isomorphisms of lattices of closed subspaces, Canad. J. Math. 36 (1984), 820-829.

[6] A. M. Gleason, Measures on the closed subspaces of a Hilbert space, J. Math. Mech. 6 (1957), 885-893.

[7] A. Guterman, C. -K. Li, and P. Šemrl, Some general techniques on linear preserver problems, Linear Algebra Appl. 315 (2000), 61-81.

[8] M. Györy, Transformations on the set of all $n$-dimensional subspaces of a Hilbert space preserving orthogonality, Publ. Math. Debrecen 65 (2004), 233-242.

[9] J. Hakeda, Additivity of ${ }^{*}$-semigroup isomorphisms among ${ }^{*}$-algebras, Bull. London Math. Soc. 18 (1986), 51-56.

[10] J. S. Lemont and P. Mendelson, The Wigner unitary-antiunitary theorem, Ann. Math. 78 (1963), 548-559.

[11] L. Molnár, An algebraic approach to Wigner's unitary-antiunitary theorem, J. Austral. Math. Soc. 65 (1998), 354-369.

[12] L. Molnár, A generalization of Wigner's unitary-antiunitary theorem to Hilbert modules, J. Math. Phys. 40 (1999), 5544-5554.

[13] L. Molnár, Generalization of Wigner's unitary-antiunitary theorem for indefinite inner product spaces, Commun. Math. Phys. 201 (2000), 120-128.

[14] L. Molnár, A Wigner-type theorem on symmetry transformations in type II factor, Int. J. Theor. Phys. 39 (2000), 1463-1466.

[15] L. Molnár, A Wigner-type theorem on symmetry transformations in Banach spaces, Publ. Math. Debrecen 58 (2000), 231-239.

[16] L. Molnár, Transformations on the set of all n-dimensional subspaces of a Hilbert space preserving principal angles, Commun. Math. Phys. 217 (2001), 409-421.

[17] L. Molnár, ${ }^{*}$-semigroup endomorphisms of $B(H)$, in: Oper. Theory Adv. Appl. 127, Birkhäuser, Basel, 2001, 465-472.

[18] L. Molnár, Orthogonality preserving transformations on indefinite inner product spaces: generalization of Uhlhorn's version of Wigner's theorem, J. Funct. Anal. 194 (2002), 248262.

[19] P. G. Ovchinnikov, Automorphisms of the poset of skew projections, J. Funct. Anal. 115 (1993), 184-189.

[20] C. E. Rickart, Isomorphic groups of linear transformations, Amer. J. Math. 72 (1950), 451-464.

[21] P. Šemrl, On isomorphisms of standard operator algebras, Proc. Amer. Math. Soc. 123 (1995), 1851-1855.

[22] P. Šemrl, Generalized symmetry transformations on quaternionic indefinite inner product spaces: An extension of quaternionic version of Wigner's theorem, Comm. Math. Phys. 242 (2003), 579-584. 
[23] P. Šemrl, Applying projective geometry to transformations on rank one idempotents, J. Funct. Anal. 210 (2004), 248-257.

[24] P. Šemrl, Orthogonality preserving transformations on the set of $n$-dimensional subspaces of a Hilbert space, Illinois J. Math. 48 (2004), 567-573.

[25] P. Šemrl, Maps on idempotents, Studia Math. 169 (2005), 21-44.

[26] P. Šemrl, Non-linear commutativity preserving maps, Acta Sci. Math. (Szeged) 71 (2005), 781-819.

[27] P. Šemrl, Maps on idempotent matrices over division rings, J. Algebra 298 (2006), 142-187.

[28] P. Šemrl, Maps on matrix spaces, Linear Algebra Appl. 413 (2006), 364-393.

[29] U. Uhlhorn, Representation of symmetry transformations in quantum mechanics, Ark. Fysik 23 (1963), 307-340.

[30] E. P. Wigner, Gruppentheorie und ihre Anwendung auf die Quantenmechanik der Atomspektrum, Vieweg, 1931. 
